\title{
Zig-zag instability of an Ising wall in liquid crystals
}

\author{
C. Chevallard, M.G. Clerc, P. Coullet, J.-M. Gilli \\ Institut Non Linéaire de Nice, UMR 6618 CNRS-UNSA, \\ 1361 Route des Lucioles, F-06560 Valbonne, France.
}

\begin{abstract}
We present a theoretical explanation for the interfacial zigzag instability that appears in anisotropic systems. Such an instability has been experimentally highlighted for an Ising wall formed in a nematic liquid crystal cell under homeotropic anchoring conditions. From an envelope equation, relevant close to the Fréedericksz transition, we have derived an asymptotic equation describing the interface dynamics in the vicinity of its bifurcation. The asymptotic limit used accounts for a strong difference between two of the elastic constants. The model is characterized by a conservative order parameter which satisfies a Cahn-Hilliard equation. It provides a good qualitative understanding of the experiments.
\end{abstract}

The zig-zag instability, undergone by straight rolls in two-dimensional extended systems like Rayleigh-Bénard convection [1] or electroconvection [2] fluid systems, have been extensively studied over the last decades. A similar zig-zag instability affecting anisotropic interfaces has recently aroused considerable interest in different fields, for example, in gas discharge system [3], crystal growth [4, rifts in spreading wax layer 5 , Ising wall in nematic and cholesteric liquid crystals $\sqrt{6}$ and chevrons layer structure of smectic liquid crystals [7]. In this letter, we present a theoretical explanation for this interfacial zigzag instability and show how it justifies the Ising wall dynamics in liquid crystal samples. Besides, the instability is experimentally characterized for a thin layer of nematic liquid crystal homeotropically anchored. From an amplitude equation, which is valid in the vicinity of the Fréedericksz transition, we derive a solvability equation that describes the interface dynamics at the onset of the bifurcation. This model is in qualitative agreement with the experimental results, even though these latter were obtained far from the Fréedericksz transition. Numerical simulations allow to strengthen the relevance of the model.

In two-dimensional extended systems, the dynamics of a straight interface can be entirely characterized by the dynamics of the interface position $P$, function that parametrizes the interface in space and time. Henceforth we consider that the variables $x$ and $y$ describe respectively the transversal and longitudinal directions of the straight interface. As a consequence of the translational invariance of the interface $\left(P \longrightarrow P+P_{o}\right)$ and of the space reflection symmetry in the tangential direction to the interface $(y \longrightarrow-y)$, the order parameter $P(t, y)$ shows a spatio-temporal evolution which is modelled by the Kuramoto-Sivashinky equation [8], as long as one assumes that, the interface only depends on the tangential direction. If one considers moreover the space reflection symmetry in the transversal direction $(P \longrightarrow-P)$, then the order parameter satisfies the following diffusion equation

$$
\partial_{t} P=\varepsilon P_{y y}
$$

When $\varepsilon$ is small (positive or negative), the order parameter dynamics is described by the asymptotic equation

$$
\partial_{t} P=\varepsilon P_{y y}+3 P_{y}^{2} P_{y y}-P_{y y y y}
$$

where $\varepsilon$ is the diffusion (antidiffusion, $\varepsilon<0$ ), $P_{y}^{2} P_{y y}$ the nonlinear diffusion and the last term the hyperdiffusion.

In the preceding expressions, we have only considered transversal perturbations to the straight interface and adiabatically eliminated the diffusive behavior in the longitudinal direction, approximations that are both correct for anisotropic systems. In isotropic systems, a straight interface can be linearly unstable $(\varepsilon<1)$ along the longitudinal direction as a result of the interface symmetry. However, in this case, nonlinear terms appear simultaneously $\left(\partial_{x} P \partial_{y y} P\right.$, and $\partial_{y} P \partial_{x y} P$ evaluated at the interface) that destabilize the solutions of eq. (2). Due to these nonlinear terms, the vertices, shown on figures 2 and 1 , will become fingers that propagate along the transversal direction [9]. Therefore all physical systems that show a zig-zag instability leading to a zigzag coarsening dynamics are anisotropic [3, 4, 5, 7].

In eq. (2) we have assumed that the nonlinear and fourth-order derivative terms saturate the instability, which imposed the signs of these two terms. Note that this equation is a continuity equation that expresses the conservation of the area enclosed by the curve $P(y, t)$ and the y-axis. This law stands from the fact that the interface connects two symmetrical states $(P \longrightarrow-P)$ and hence, when one part of the interface moves in one direction, another part moves simultaneously in the opposite direction. Moreover, this equation is variational,i.e. it can be rewritten in the following form

$$
\partial_{t} P=-\frac{\delta \mathcal{F}}{\delta P}, \quad \mathcal{F}[P]=\int d y\left\{\varepsilon \frac{P_{y}^{2}}{2}+\frac{P_{y}^{4}}{4}+\frac{P_{y y}^{2}}{2}\right\}
$$

where the free energy $\mathcal{F}$ only depends on the order parameter derivatives. Introducing the variable $\Lambda \equiv P_{y}$, which describes the local tilt of the interface with respect to the original orientation of the straight interface, the latter equation is reduced to 


$$
\partial_{t} \Lambda=\partial_{y y}\left(\varepsilon \Lambda+\Lambda^{3}-\Lambda_{y y}\right)
$$

This equation is the well-known Cahn-Hilliard equation [12], which describes the phase separation dynamics in conservative systems. The dynamical behavior of this equation has been studied by many authors (see for example [13, 14] and the references therein). It is noteworthy that the relevant conservation law for the dynamics is actually

$$
M=\int \Lambda(y, t) d y
$$

and not the integral of $P$, as a consequence of the translational invariance. When $M$ is zero, the dynamical evolution, deduced from the theoretical analysis, is in good quantitative agreement with the experiments, as we shall see later. A wave number of order $\sqrt{-\varepsilon / 2}$ arises initially in the system. Later on, the system exhibits a coarsening dynamics (see fig. 2) during which the wavelength increases. New periodic solutions emerge with a logarithmically slow growth rate 14].

To find the steady solutions, one has to minimize the free energy taking into account the area conservation. This can be done by considering a Lagrange multiplier $(\lambda)$. Hence, the free energy takes the form [15]

$$
\mathcal{G}[\Lambda]=\int d y\left\{\varepsilon \frac{\Lambda^{2}}{2}+\frac{\Lambda^{4}}{4}+\frac{\Lambda_{y}^{2}}{2}+\lambda \Lambda\right\}
$$

The family of solutions that minimize this free energy satisfies the differential equation

$$
\Lambda_{y y}=\lambda+\varepsilon \Lambda+\Lambda^{3}
$$

Obviously such solutions also satisfy eq. (41). The global minimum of the free energy $\mathcal{G}$ is either the homoclinic solution (which is an interface with three facets) or the constant solution. This one, as well as the facets, can be any of the local minima of the potential $V=\lambda \Lambda+$ $\varepsilon \frac{\Lambda^{2}}{2}+\frac{\Lambda^{4}}{4}$. We remark that these solutions have different Lagrange multiplier values.

Let's consider now an anisotropic nematic liquid crystal sample that exhibits experimentally an interfacial zigzag instability, as emphasized below. This instability will be theoretically described in the framework of the nonlinear elasticity theory of liquid crystals.

In the experiments, we used a cyanobiphenyl compound $(5 \mathrm{CB})$ whose anisotropic physical properties at $25^{\circ} \mathrm{C}$ are: elastic constants $K_{1}=6.3, K_{2}=4.1, K_{3}=8.4$ (10 $10^{-7}$ dynes); dielectric anisotropy $\epsilon_{a}=11.3$; diamagnetic anisotropy $\chi_{a}=1.142\left(10^{-7} \mathrm{~cm}^{3} g^{-1}\right)$; rotational viscosity $\gamma_{1} \sim 10^{-2} \mathrm{~Pa}$.s.

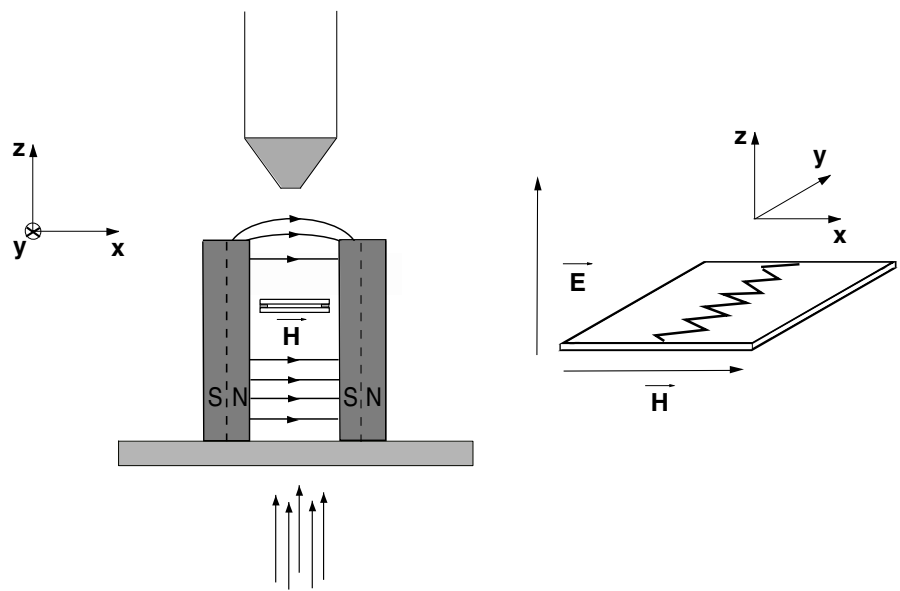

FIG. 1: Experimental set-up: the sample of liquid crystal is placed between two permanent magnets which determine the homogeneous magnetic field $\vec{H}$. The physical phenomena are observed through a polarizing microscope.

The samples are made of two glass plates separated by thin mylar spacers that determine the cell thickness (between 50 and $250 \mu \mathrm{m}$ ). The glass surfaces are treated with lecithin to provide homeotropic anchoring. The sample is subjected to a sinusoidal vertical electric field $\vec{E}=E \vec{e}_{z}\left(V_{e f f} \sim 0-9 V\right)$ with a high frequency $(\sim 5 k \mathrm{~Hz})$ in order to avoid charge injection or electroconvection phenomena. Moreover, two permanent magnets induce a homogeneous horizontal magnetic field in the sample. The field magnitude can be changed by bringing the magnets nearer or farther. Its maximum value is about 0.55 Tesla.

The application of a horizontal magnetic field can induce, for a high enough magnitude, a partial reorientation of the bulk molecules along the field direction. Due to the twofold degeneracy of the bifurcated state, domains of opposite orientation may be created [10]. The interface between a pair of domains is called Ising wall [11]. An Ising wall in a splay-bend configuration is formed by using the flux lines curvature in a region where the field is inhomogeneous (see fig. 1). Then, it is quenched into the area between the two magnets, where the field is homogeneous. Its width is determined by the distance to the threshold of the Fréedericksz transition and can be modified by variations of the fields magnitude.

The interface behavior is observed through a polarizing microscope (see fig. 1). Video films or numerical images (see fig. 2) can be registered thanks to a $3 \mathrm{CCD}$ camera placed on the top of the microscope.

The experiments have been carried out far from the Fréedericksz threshold, since near the transition any little imperfection on the parallelism between the sample and the magnetic field plane makes the wall drift towards one edge of the cell. 


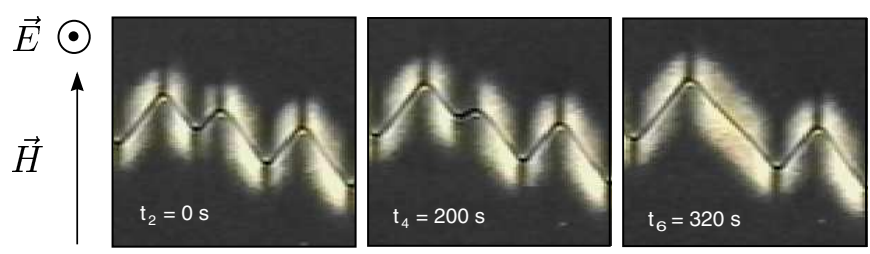

FIG. 2: Spinodal decomposition of the interface observed through a microscope with crossed polarizers.

The splay-bend Ising wall is ever unstable, for the control parameters that we used experimentally, when it is thrust into the homogeneous area of the magnetic field. Initially, the interface develops an instability characterized by a well-defined wavelength, which is determined by the experimental parameters. Later on, the sinusoidal interface becomes an angled line composed of pieces of wall turned with angles $\pm \Psi_{0}$ (see fig. 2). Two adjacent pieces, whose orientations are opposite, are connected by a region of strong curvature of the line that we called kink. The dynamics consists then in reassembling domains of even orientation, the angle of the "zig" and "zag" facets staying unchanged (coarsening dynamics). This process occurs thanks to annihilations of kinks and without characteristic lengthscale. Actually, the averaged domain size increases regularly in time (see fig. 2). The dynamics, which tends to separate the zig and zag facets, is the one-dimensional counterpart of the spinodal decomposition dynamics observed in conservative binary mixtures 12.

The instability is triggered by the elastic anisotropy of the liquid crystal whose influence is emphasized in the wall. Indeed, the involved distortions depend on the orientation of the interface with respect to the magnetic field direction. For most of the usual compounds, the energy cost of a wall aligned with the field (twist wall) is lower than the one of a wall perpendicular to the field (splay-bend wall) since $K_{2}<K_{1}$. Consequently, the system (splay-bend wall) reduces its energy by changing the direction of the wall. A global rotation of the interface can not occur in an infinite medium. Therefore, the interface is forced to rotate locally and is divided into facets turned with opposite angles $\pm \Psi_{0}$. These angles, whose values are determined by the control parameters (see fig. 3), result from several effects. More precisely, the rotation is favored by the elasticity but is made difficult by the resulting elongation of the interface along its original direction and by the escape of the molecules from the vertical plane containing the magnetic field. The local reorientation generates many defects (kinks), which will eventually disappear since this leads to a decrease in energy.

The experiments have been performed far from the

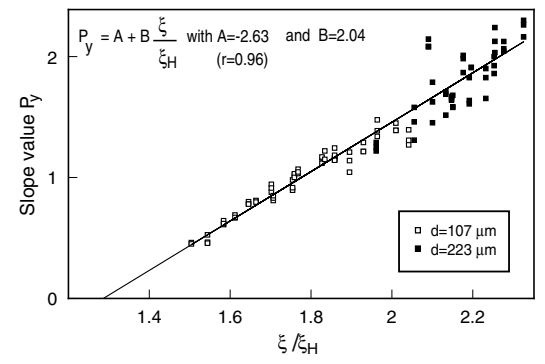

FIG. 3: Experimental curve giving the slope $P_{y}$ of the interface versus the dimensionless ratio $\frac{\xi}{\xi_{H}}$ ( $\xi$ is the electromagnetic coherence length and $\xi_{H}$ the magnetic coherence length).

Fréedericksz transition. However, for the sake of simplicity, the theoretical approach has been developed near this transition. At the onset of the bifurcation, the system is described by the two-dimensional Landau equation

$$
\partial_{T} Z=\varepsilon Z-b Z^{3}+\left(K_{1} \partial_{x}^{2}+K_{2} \partial_{y}^{2}\right) Z
$$

where $\varepsilon \equiv-\epsilon_{a} E^{2}-K_{3} \frac{\pi^{2}}{d^{2}}+\chi_{a} H^{2}, b \equiv \frac{1}{2}\left(K_{1}-\frac{3}{2} K_{3}\right) \frac{\pi^{2}}{d^{2}}-$ $\frac{3}{4} \epsilon_{a} E^{2}+\frac{3}{4} \chi_{a} H^{2}, T=t / \gamma_{1}$, and the director takes the form $\vec{n}=\left(Z \cos \left(\frac{\pi z}{d}\right), 0,1-\frac{Z^{2}}{2} \cos ^{2}\left(\frac{\pi z}{d}\right)\right)$. This equation contains wall-type solutions. When both diffusion constants $\left(K_{1} / \gamma_{1}\right.$ and $\left.K_{2} / \gamma_{1}\right)$ are of the same order of magnitude, these solutions are always stable. In order to explain the appearance of the interfacial instability, one has to consider that $K_{2}$ is much smaller than the other constants $K_{1}$ and $K_{3}\left(K_{2} \sim \varepsilon\right)$. Then, the solution $Z=0$ is marginal with respect to perturbations in the $y$-direction. Note that the interface identifies with the center of the Ising wall, which is a curve where $Z=0$ (homeotropic state). Hence, higher-order terms must be considered in order to study the stability of the interface, and the dynamical equation reads now

$$
\begin{aligned}
\partial_{T} Z= & \varepsilon Z-b Z^{3}+\left(K_{1} \partial_{x}^{2}+K_{2} \partial_{y}^{2}\right) Z+\frac{K_{1}^{2}}{a} \partial_{x^{2} y^{2}} Z \\
& +\frac{K_{1}^{3}}{a^{2}} \partial_{x^{2} y^{4}} Z+\frac{3}{4} K_{3}\left(Z\left(\partial_{y} Z\right)^{2}-\frac{Z^{2}}{2} \partial_{y y} Z\right)(9)
\end{aligned}
$$

where we have considered the asymptotic limit $Z \sim \varepsilon^{1 / 2}$, $\partial_{y}^{2} Z \sim \mu \varepsilon^{1 / 2}, \partial_{x}^{2} Z \sim \varepsilon^{3 / 2}, K_{1} \sim K_{3} \sim 1$, and $K_{2} \sim \varepsilon$, with $\varepsilon \ll \mu \ll 1, a \equiv \epsilon_{a} E^{2}+K_{3} \frac{\pi^{2}}{d^{2}}$ and $\mu \equiv\left(\frac{K_{2}}{\varepsilon}-\frac{2 K_{1}}{5 a}\right)$. Then, the expression of the local director is given by

$$
\vec{n}=\left(\begin{array}{c}
n_{x} \\
n_{x} \\
n_{z}
\end{array}\right)=\left(\begin{array}{c}
Z \cos \left(\frac{\pi z}{d}\right) \\
\left(\frac{K_{1}}{a} \partial_{x y} Z+\frac{K_{1}^{2}}{a^{2}} \partial_{x y^{3}} Z\right) \cos \left(\frac{\pi z}{d}\right) \\
1-\frac{Z^{2}}{2} \cos ^{2}\left(\frac{\pi z}{d}\right)
\end{array}\right)
$$

In this asymptotic limit, the Ising wall solution reads $Z=\sqrt{\varepsilon / b} \tanh \left(\sqrt{\varepsilon / 2 K_{1}}\left(x-x_{o}\right)\right)$. In order to describe 


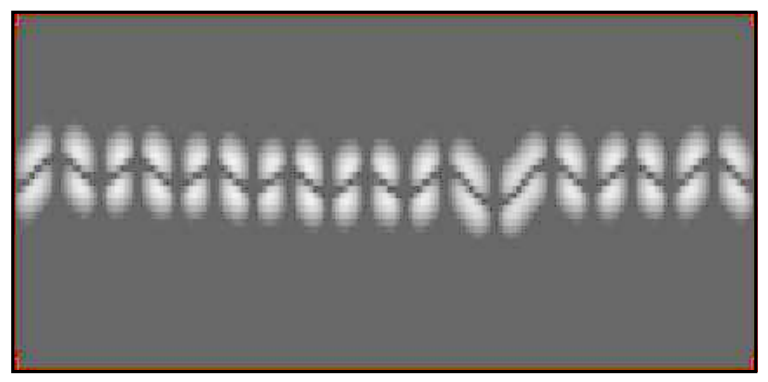

FIG. 4: Numerical simulation of the eq.(8) for a high anisotropy of elasticity : $\frac{K_{2}}{K_{1}}=0.056, K_{3}=1, a=1$. The picture shows the Y-component of the director in the system.

the dynamical behaviour of the wall under perturbations, we introduce the following ansatz

$Z(x, P(y, T)) \equiv \sqrt{\frac{\varepsilon}{b}} \tanh \left(\sqrt{\frac{\varepsilon}{2 K_{1}}} x-P(y, T)\right)+w(x, P)$

where $w(x, P)$ is a small perturbative term. One can show that, $P$ satisfies the following solvability equation

$$
\partial_{T} P=D_{1} P_{y y}+D_{2} P_{y}^{2} P_{y y}-D_{3} P_{y y y y}
$$

with $D_{1} \equiv \mu \varepsilon=\left(K_{2}-\frac{2 K_{1} \varepsilon}{5 a}+\frac{3 K_{3} \varepsilon}{40 b}\right), D_{2} \equiv \frac{48 K_{1}^{2}}{7} \frac{\varepsilon}{a^{2}}$, $D_{3} \equiv \frac{2 K_{1}^{2}}{5} \frac{\varepsilon}{a^{2}}$.

Thus, the characteristic time-scale of the interface dynamics $\left(\mu^{-1} \varepsilon^{-1}\right)$ is smaller than the time-scale of the interface formation $\left(\varepsilon^{-1}\right)$, which is consistent with experimental observations. The expression of $D_{1}$ indicates that the elastic anisotropy can give rise to the instability $\left(D_{1}<0\right)$. Numerical simulations of eq. (9) confirms the latter calculations (see fig. (1).

As a consequence of the finite size of liquid crystal sample, one experimentally observes a global rotation of the interface, which is superimposed to the coarsening dynamics. This favours one orientation of the domains, but does not change the angle of the facets.

In conclusion, we have presented a theoretical explanation of the interfacial zig-zag instability that appears in anisotropic systems. The instability of an Ising wall formed in a nematic liquid crystal has been more specifically investigated, both experimentally and theoretically. Close to the Fréedericksz transition, this instability is described by the Cahn-Hilliard equation, and leads to the facets separation and coarsening dynamics. The asymptotic limit used in the derivation of this model emphasizes the role of the elastic anisotropy. Such a limit contains all the ingredients required to give an adequate description of the experimental observations.

The authors acknowledge S. Thiberge for fruitful discussions. One of us P.C. thanks the support of the "Institut Universitaire de France" and M.C. thanks he support of Cátedra Presidencial. The simulation software developed at the laboratory INLN in France has been used for all the numerical simulations presented in this paper.

[1] MAnneville P. and PIQUEMAL J.-M., Phys. Rev. A , 28 (1983) 1774; CROSS M. C. and HOHENBERG P. C., Rev. Mod. Phys., 65 (1993).

[2] RIBOtTA R., JOETS A., and LEI L., Phys. Rev. Lett. 56 (1986) 1595; BODENSCHATZ E., KAISER M, KRAMER L., PESCH W., WEBER A., ZIMMERMANN W., New Trends in Nonlinear Dynamics and Pattern-Forming Phenomena, edited by P. COULLET and P. HUERRE (Plenum Press, New York) 1990.

[3] ASTROV Yu. A., AMMELT E. and PURWINS H.G., Phys. Rev. Lett., 78 (1997) 3129.

[4] GOLOVIN A. A., DAVIS S. H., NEPOMNYASHCHY A. A., Physica D, 122 (1998)202.

[5] RAGNARSSON R., FORD J.L., SANTANGELO C. D. and BODENSCHATZ E., Phys. Rev. Lett., 76 (1996) 3456.

[6] CHEVAllard C., CleRC M., COUllet P.and GILLI J.M., Eur. Phys. J. E., 1 (2000) 179.

[7] LIMAT L., Europhys. lett., 44 (1998) 205.

[8] KuRAmoto Y. , TSUZUKI T., Prog. Theor. Phys., 55 (1976) 356; SIVASHINSKY G.I., Acta Astronautica, 4 (1977) 1177.

[9] CHEVALLARD C. and CLERC M., in preparation.

[10] HELFRICH W., Phys. Rev. Lett., 21 (1968) 1518.

[11] BULAEVSKII L. N. and GINZBURG V. L., Zh. Eksp. Teor. Fiz., 45 (1963) 772. [Sov. Phys. JETP, 18 (1964) 530]; COULLET P., LEGA J., HOUCHMANZADEH B. and LAJZEROWICZ J., Phys. Rev. Lett., 65 (1990) 1352.

[12] CAHN J. W., HILliarD J. E., J. Chem. Phys., 28 (1958) 258.

[13] KAWASAKI T., MUNAKATA T., Prog. Theor. Phys. , 74 (1988) 656; KAWASAKI T., OHTA T., Physica A, 11 (1982) 573.

[14] Alikakos N., BATES P. W., FUSCO G., J. Diff. Eqs., 90 (1990) 81; BATES P. W., XUN J. P., J. Diff. Eqs., 111 (1990) 421.

[15] ELLIOT C. M., FRENCH D., IMA Journal Appl. Math., 38 (1987) 97; EILBECK J.C., FURTER J.E., GRINFELD M., Phys. lett. A, 135 (1989) 272. 